\title{
FORMACIÓN DEL PROFESORADO UNIVERSITARIO EN LAS COMPETENCIAS DOCENTES
}

\author{
Antonio Medina Rivilla ${ }^{1}$ \\ Universidad Nacional de Educación a Distancia - España \\ Grupo de investigación HISULA \\ amedina@edu.uned.es \\ $\mathbf{M}^{\mathrm{a}}$ Concepción Domínguez Garrido 2 \\ Universidad Nacional de Educación a Distancia - España \\ cdominguez@edu.uned.es \\ Fernando Ribeiro Gonçalves ${ }^{3}$ \\ Universidade do Algarve - Portugal \\ Grupo de investigación HISULA \\ frgcasa@gmail.com
}

Recepción: 02/07/2010

Evaluación: 27/04/2011

Aceptación: 10/10/2011

Artículo de Reflexión

\section{RESUMEN}

La investigación realizada sintetiza las aportaciones de varios proyectos orientados a la formación, el desarrollo profesional y al conocimiento de las competencias más valiosas a dominar por el profesorado universitario. Se construye un mapa de las competencias docentes más estimadas por

1 Antonio Medina Rivilla, Doctor en Pedagogía y Licenciado en Psicología. Catedrático de Didáctica y Organización escolar, Profesional de Educación a Distancia en UNED- España. Experto en Diseño de programas para el desarrollo de conferencias en entornos educativos y de las organizaciones. Doctor y Profesor Honoris Causa, Instituto Universitario Italiano de Rosario (IUNIR). Argentina.

2 Dra. $\mathrm{M}^{\mathrm{a}}$ Concepción Domínguez Garrido, Catedrática de universidad de Ciencias Sociales. Departamento de Didáctica, Organización Escolar y Didácticas Especiales. Profesional de Educación a Distancia en UNED - España.

3 Fernando Ribeiro Gonçalves, Doctor en Ciencias de la Educación. Catedrático Universidad do Algarve - Portugal. Presidente de RIAICES (Red Iberoamericana en investigación de la calidad de la Educación Superior). Vicepresidente de la Sociedad Portuguesa de Ciencias de la Educación. 
expertos, una amplia muestra de docentes y tutores, a partir del cual se diseña un modelo para impulsar la formación del profesorado universitario y la consolidación de la identidad profesional, mediante la integración de métodos cuantitativos y cualitativos.

Se destacan dos grandes modalidades de competencias: Las ligadas al dominio de las claves del EEES, la sociedad del conocimiento, la comunicación, investigación e innovación de la docencia, y las ligadas a la mejora del proceso formativo: identidad profesional, planificación, tutoría, sistema metodológico, diseño de medios, evaluación; que han de integrar la teoría y práctica docente en las instituciones formativas.

Palabras clave: Revista Historia de la Educación Latinoamericana, desarrollo profesional, competencias docentes, competencias discentes, clima institucional formativo, integración metodológica, complementariedad de los modelos formativos.

\title{
UNIVERSITY TEACHER TRAINING IN TEACHING SKILLS
}

\author{
Antonio Medina Rivilla \\ Universidad Nacional de Educación a Distancia - España \\ Research group HISULA \\ amedina@edu.uned.es \\ $\mathbf{M}^{\mathrm{a}}$ Concepción Domínguez Garrido \\ Universidad Nacional de Educación a Distancia - España \\ cdominguez@edu.uned.es \\ Fernando Ribeiro Gonçalves \\ Universidade do Algarve - Portugal \\ Research group HISULA \\ frgcasa@gmail.com
}

\begin{abstract}
The research summarizes the contributions of research projects aimed at training, professional development and knowledge of the most valuable skills mastered by the faculty. We build a map of the teaching competencies most valued by experts, a large sample of teachers and tutors, at the end of the cycle, from which a model is designed to promote teacher training college and the consolidation of professional identity, or through the integration of quantitative and qualitative methods. It highlights two major forms of competence: domain linked to the keys of the EHEA, the society
\end{abstract}


of knowledge, communication, research and innovation in teaching and those linked to improving the teaching process: professional identity, mentoring, planning, methodological system, media design, evaluation, they have to integrate teaching theory and practice in educational institutions.

Key words: Journal of Latin American Education History, professional development, teaching skills, competencies learners, institutional climate, training, methodological integration, complementarity of the training models.

\title{
FORMAÇÃO DO PROFESSORADO UNIVERSITÁRIO NAS COMPETÊNCIAS DOCENTES
}

\author{
Antonio Medina Rivilla \\ Universidad Nacional de Educación a Distancia - España \\ Grupo de pesquisa HISULA \\ amedina@edu.uned.es \\ $\mathbf{M}^{\mathbf{a}}$ Concepción Domínguez Garrido \\ Universidad Nacional de Educación a Distancia - España \\ cdominguez@edu.uned.es \\ Fernando Ribeiro Gonçalves \\ Universidade do Algarve - Portugal \\ Grupo de pesquisa HISULA \\ frgcasa@gmail.com
}

\section{RESUMO}

A pesquisa realizada sintetiza as contribuições de vários projetos, orientados à formação, o desenvolvimento profissional e ao conhecimento das competências mais valiosas para o professorado universitário. Construiuse um mapa das competências docentes mais estimadas por expertos, uma ampla mostra de docentes e tutores, a partir do qual se desenha um modelo para impulsionar a formação do professorado universitário e a consolidação da identidade profissional, mediante a integração de métodos quantitativos e qualitativos.

Destacam-se duas grandes modalidades de competências: as ligadas ao domínio das chaves do EEES, a sociedade do conhecimento, a comunicação, pesquisa e inovação da docência, e as ligadas à melhora do processo formativo: identidade profissional, planificação, tutoria, sistema metodológico, desenho de meios, avaliação; que iriam de integrar a teoria e prática docente nas instituições formativas. 
Palavras-chave: Revista História da Educação Latino-americana, desenvolvimento profissional, competências docentes, competências discentes, clima institucional formativo, integração metodológica, complementaridade dos modelos formativos.

\section{INTRODUCCIÓN}

La docencia universitaria es un proceso formativo, renovado por las directrices del EESS (Espacio Europeo de Educación Superior) y de un mundo en continuo cambio, excesivas tensiones: económicas, socio-políticas, culturales, laborales, tecnológicas, etc., que demanda de la institución universitaria una "Nueva visión y más opciones transformadoras". Ante tal complejidad de cambios es necesaria la recuperación de lo más valioso de la historia académica y de la cultura universitaria, por lo que nos hemos de preguntar ¿Qué docente y académico universitario se requiere actualmente? ¿Qué relación y armonización de este académico con los restantes del sistema educativo y singularmente con los del nivel de educación secundaria? Desde este contexto y desde este texto - pretexto: ¿Qué modelos, programas y formas de actualizar al profesorado universitario estamos desarrollando. ¿Qué espera aportar este artículo a un problema tan complejo?.

Confiamos ofreceralgunas oportunidades yactualizarotras investigaciones ya consolidadas y retomadas en varias obras Medina y $\operatorname{cols}^{4}$ y la síntesis de las aportaciones del equipo de investigación e innovación educativa desde 2006 a 2010, entre ellas, Medina, Domínguez y Sánchez ${ }^{5}$. Presentamos a continuación una síntesis de las líneas desarrolladas, siendo conscientes de la complejidad del problema de investigación que continuamos profundizando.

El núcleo problemático que abordamos, es identificar las modalidades, programas y aspectos esenciales que el profesorado ha de dominar para el desempeño de la docencia e investigación universitaria en el siglo XXI, a la vez que encontrar las claves para que esta plena formación les facilite

${ }^{4}$ MEDINA, Antonio. (2003): Modelos de evaluación de la calidad de las instituciones universitarias. Madrid, Universitas, p. 86.

MEDINA, Antonio y cols. (2006): Formación y evaluación de las competencias del profesorado. Madrid, Ministerio de universidades e investigación. p. 79.

MEDINA, Antonio; SEVILLANO, María Luisa; DE LA TORRE, Saturnino. (Coords.) (2009): La Universidad del siglo XXI. Madrid, Universitas. p. 215. MEDINA, Antonio; DOMÍNGUEZ, María Concepción; SÁNCHEZ, Cristina. (2010): "Formación de las competencias socio-profesionales desde la función tutorial", en: III Jornadas de Redes. Madrid, UNED. p. 384.

5 MEDINA, Antonio; DOMíNGUEZ, María Concepción; SÁNCHEZ, Cristina. (2009): "Formación de las competencias de los discentes, mediante un diseño integrado de medios", en: Revista Eccos, Vol.10, No. 2, pp. 327-360. 
el logro de un estilo académico, que promueva el óptimo desarrollo y la formación en la docencia para el resto de los docentes del sistema educativo.

La pregunta central a la que pretendemos responder es ¿ Hemos construido algún modelo valioso y representativo que facilite la formación integral del profesorado universitario y le sitúe como el experto en el saber académico, las prácticas docentes y el creador de una línea de investigación e innovación de la docencia universitaria?, que completamos con las siguientes: ¿El profesorado universitario ha de incorporar a su investigación el compromiso con la mejora continua de su docencia y de la formación en y desde su conocimiento como profesional competente? ¿Es factible implicar al profesorado universitario en la transformación reflexiva y transformadora de su docencia?

El profesorado universitario ha estado centrado en realizar una docencia orientada al saber académico, necesario y cada vez más globalizado y abundante, pero insuficiente para dar respuesta a los nuevos retos de la sociedad del conocimiento, las demandas de los empleos y los cambios exponenciales en los estilos y modos de desempeñar la vida laboral, impregnada por la utilización de las TIC, así como la incidencia de las redes sociales y la continua transformación de la cultura, las organizaciones y los múltiples escenarios socio - laborales, con abundantes culturas y modos de resolver los problemas.

El diagnóstico evidencia una alta complejidad social e institucional, afectada por situaciones mundiales cada vez más convulsas y la urgencia de una auténtica profesionalización de los docentes y discentes. Esta gran diversidad de problemas nos ha llevado a conformar un grupo de investigación e innovación de la docencia universitaria ${ }^{6}$ (proyecto MOEES, 2006, 2007); la consolidación de una línea de Congresos ${ }^{7}$ (DIPLAES, Abril de 2008, con edición de la obra: Universidad del Siglo XXI, ante el EEES) ${ }^{8}$; (INNOVADOC, 2009, Investigación e innovación de la docencia universitaria en el EEES, 2010) y la constitución y desarrollo de la RED ${ }^{9}$ (RIAICES, 2010) que avanza en la edición de un nuevo congreso.

6 Proyecto de desarrollo futuro en el Espacio Europeo de Educación Superior. (2007). Madrid, Ed. Ministerio de Universidades e Innovación (edición on line). pp. 25-86.

7 Congreso Internacional sobre las nuevas bases para el Diseño de los Planes de Estudio en el EEES y su incidencia en el diseño educativo. 2009. Madrid, Ed. Universitas. pp. 17-105.

8 Congreso Internacional sobre Investigación e Innovación de la Docencia universitaria en el EEES. (2010). Madrid, Ramón Areces. pp. 25-105.

9 Red Iberoamericana de Investigación de la Calidad de la Educación Superior. (2010). RIAICES, (actualmente, nueva URL 2012. 
Así, las publicaciones coordinadas individualmente o en colaboración ${ }^{10}$ (1997, 2003, 2006, 2007, 2008, 2009, 2010) representan una línea consolidada que evidencia que la formación y mejora de la docencia universitaria es esencial y hemos de cuestionarnos los modelos de actualización y desarrollo profesional del profesorado universitario, constituido en el principal protagonista de las transformaciones de la cultura y las prácticas docentes en las universidades.

\section{1. ¿Qué modelo de desarrollo profesional del docente universitario estamos construyendo?}

El profesorado universitario se consolida como el artífice del liderazgo formativo para todos los docentes del sistema educativo, dado que en la universidad, así lo constatan los nuevos másteres en el EEES, se ha convertido en la legitimadora de la innovación de la educación, la anticipadora de los retos de la sociedad del conocimiento y la creadora de la nueva cultura que demanda esta sociedad. Por ende, hemos generado y compartido modelos de desarrollo profesional con otros investigadores $\mathrm{y}$ asociaciones de formación del profesorado como ${ }^{11}$ ISATT, ${ }^{12} \mathrm{ECER}$, Asociación RIAICES; una de sus principales tareas es mejorar la calidad de las universidades mediante: La formación y actualización integral del profesorado universitario. Estas modalidades formativas se han iniciado y consolidado en la última década (2000-2010) avanzando en el dominio de la docencia universitaria como profesión, práctica y tarea plenificadora para cada universitario y la sociedad en su conjunto.

Las investigaciones nos propician algunas modalidades formativas ya consolidadas, que sintetizamos en los escenarios, que se detallan a continuación: Modalidad formativa basada en el autoconocimiento de las experiencias prácticas relevantes y la consolidación de la narrativa de vida,

${ }^{10}$ MEDINA, Antonio y RODRÍGUEZ, Ana. (1997): "Programas de formación inicial del profesorado", en: Actas $V$ Congreso interuniversitario de organización de Instituciones educativas. Madrid, 10-13 de noviembre. pp.710740; MEDINA, Antonio. (2003): Óp., cit, pp. 85-97; MEDINA, Antonio y cols. (2006): Óp., cit, pp. 25- 86; MEDINA, Antonio y DOMÍNGUEZ, María Concepción. (2007): "La formación de las competencias profesionales mediante el empleo de la plataforma de investigación en docencia universitaria”. IV Jornadas ICE. Alicante 4-5 de julio; MEDINA, Antonio y DOMÍNGUEZ, María Concepción. (2008): Los procesos reflexivos como base de la formación del profesorado, en: CARDONA, José. Cuestiones en torno a la formación y desarrollo profesional de los profesores. Madrid, Sanz y Torres, pp. 157-182; MEDINA, Antonio (ed.) (2009): "Formación y desarrollo de las competencias básicas". Madrid, Universitas, pp. 5-96; DOMÍNGUEZ, María Concepción, MEDINA, Antonio y CACHEIRO, María Luz. (2010): Investigación e innovación de la docencia universitaria en el EEES. Madrid, Editorial Universitaria. Ramón Areces, p. 30.

${ }^{11}$ International Study Association on Teachers and Teaching. ISATT (Acrónimo de la asociación).

12 European Conference on Educational Research. ECER (Acrónimo de la asociación). 
como la base del conocimiento, mejora y transformación del saber y hacer profesional; Identificación de la aportación del paradigma del pensamiento del profesorado, complementado con el "Interpensamiento compartido del profesorado", avanzando en el autodesarrollo personal y profesional e implicando a los colegas en esta mejora continua; Superar las limitaciones de la formación inicial de los docentes universitarios y plantear programas adaptados a la implicación y socialización de los jóvenes profesionales en su futuro desarrollo; La formación apoyada en el trabajo colaborativo entre docentes jóvenes y experimentados, y entre cuantos generan una línea de avance comprometido con el significado de la acción formativa en la universidad; Formación basada en el conocimiento, dominio y transformación continua de las competencias docentes, consideradas necesarias para avanzar en el desarrollo de las competencias discentes.

La representación gráfica de esta línea de investigación e innovación consolidada en este periodo, con la realización de estudios focalizados en la creación de un modelo de armonía, corresponsabilidad y reflexiónnarrativa semestral, del equipo (Medina, Domínguez y Sánchez 2006-2010) y docentes tutores colaboradores que, se ha consolidado en el escenario y las claves formativas que esquemáticamente presentamos:

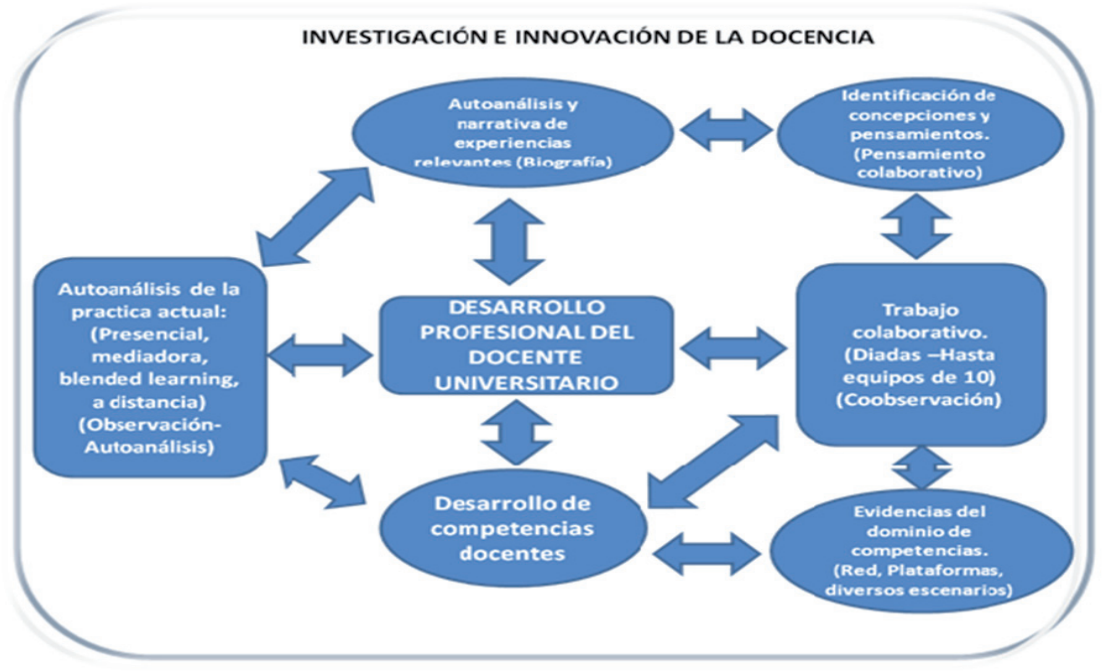

\section{Construcción y claves para un modelo de desarrollo profesional en tiempos complejos}

Le Boterf ${ }^{13}$ subraya la necesidad de armonizar y dominar competencias, junto a la necesidad de ser un docente, formador y un práctico como profesional competente. Esta precisión nos cuestiona: ¿Cuál es la diferencia entre un

${ }^{13}$ LE BOTERF, Guy. (2010): Construire les compétences individuelles et collectives : agir et réussir avec compétence. Paris, Eyrolles, p 17. 
docente que avanza en el dominio de las competencias docentes y aquel que comprometiendo la lógica de las competencias, se siente activo y colabora de modo competente? Así pues, el modelo de formación que trabajamos supera la tendencia del dominio de competencias y contiene las claves para comprender la "lógica de las competencias docentes" que el profesorado universitario ha de alcanzar y hacer realidad en la docencia habitual y ordinaria.

Este modelo sintetiza las necesidades derivadas de la formación para un óptimo desarrollo de las competencias con la naturaleza reflexivoindagadora que ha de caracterizar cada práctica, emergiendo de ella lo más valioso, a saber: ¿Qué ha transformado nuestra práctica en un proceso de desarrollo profesional? ¿En nuestra historia profesional qué tipo de experiencias, modalidades formativas y redes sociales, comunidades de aprendizaje, nos facilitan el avance profundo como docentes universitarios?

$\mathrm{Si}$ a la reflexión rigurosa y personalizada en torno a nuestra propia práctica incorporamos las necesidades de la investigación e innovación de ella, encontraremos el programa formativo más fecundo en el que hemos trabajado, a fin de lograr una nueva vitalidad como docentes. La narrativa autobiográfica, el autoaprendizaje experiencial, la emergencia de concepciones y principios de nuestra práctica, unidas a la identificación de las teorías implícitas, han de ampliarse con la reflexión colaborativa y el conocimiento y avance en las competencias profesionales más relevantes.

Entre otras investigaciones el proyecto (MOEES) y el congreso (INNOVADOC), así como los trabajos de los colegas Zabalza ${ }^{14}$, De la $\mathrm{Hoz}^{15}$, Villar y Alegre ${ }^{16}$, constatan que la formación de las competencias docentes es el núcleo esencial de la capacitación de los docentes universitarios y hemos de trabajar en construir con su colaboración el MAPA MÁS REPRESENTATIVO. Las intersecciones de las competencias que evidencian estos estudios, subrayan entre las más relevantes las siguientes:

- Innovación de la docencia, en sí misma creadora de nuevo saber.

- Implicación en la sociedad del conocimiento.

- Comunicativa (Interacción didáctica).

- Planificación del conocimiento científico y de la docencia.

${ }^{14}$ ZABALZA, Miguel Ángel. (2006): "Competencias docentes del profesorado universitario". Calidad y desarrollo profesional. Madrid, Narcea, Ed. Multimedias, p. 39.

${ }^{15}$ DE LA HOZ, Gustavo. (2009): Los médicos y la educación médica: una mirada desde la teoría sobre la formación de competencias socio-profesionales en el profesorado universitario. Barranquilla, Universidad Libre, p 58.

${ }^{16}$ VILLAR, Luis y ALEGRE, Olga. (2009):"Investigación evaluativa de un título propio: Mater EDECAR en la diversidad”, en: Revista Enseñanza, Vol. 27, pp. 17-49. 
- Desarrollo del sistema metodológico y estrategias metodológicas.

- Valoración de las dimensiones europeas de la Educación Superior.

- Trabajo tutorial.

- Identidad profesional.

- Diseño de medios.

- Organización de los aprendizajes.

- Evaluadora.

\section{Modelo de organización y mejora de las competencias en el espacio europeo de educación superior}

El desarrollo profesional del profesorado universitario ha de lograrse al avanzar en el dominio y mejora de las competencias empleando las modalidades anteriores y convirtiendo su práctica en una acción docente indagadorainnovadora, que aplica los métodos y técnicas pertinentes, a la vez que la convierte en un escenario reflexivo colaborativo, así hemos de proceder de la narrativa, a la auto y coobservación de la práctica, con la emergencia y autocrítica conjunta entre docentes y estudiantes aplicada en estrecha interacción.

La formación en docencia, se convierte en la actividad esencial del profesorado ante los retos de las TIC, la interculturalidad, la transversalidad de los saberes y los auténticos retos socio-laborales para los estudiantes. Esta transformación permanente del sentido del saber y de las disponibilidades tecnológicas han de ser abordadas por el profesorado como una exigencia profesional, dado que cada docente ha de aspirar a indagar su práctica, a aprender de ella y a compartir con los colegas el gran desafío de la docencia en una mejora continua.

El sentido del cambio exponencial ha de vivirse y entenderse, conscientes de que es el profesorado el que ha de asumir un gran liderazgo en situaciones de complejidad e incertidumbre y éstas necesitarán para ser superadas un nuevo marco de saberes y especialmente, una cultura de los procesos de enseñanza-aprendizaje, que convierta a los docentes en agentes transformadores, anticipadores y críticos.

Diseño del proceso de investigación seguido de objetivos

- Identificar las competencias docentes más relevantes para desempeñar los procesos de enseñanza-aprendizaje en la universidad.

- Generar un modelo de formación de docentes, basado en el desarrollo del conjunto de competencias identificadas.

- Aplicar la integración de métodos para evidenciar las competencias más relevantes a desarrollar por el profesorado.

- Constatar la necesidad de consolidar un conjunto integrado de competencias docentes como base para desarrollar las discentes. 
- Aportar nuevas líneas para el desarrollo profesional de los docentes, mediante la complementariedad de narrativas/autobiográficas, y la coobservación entre colegas para avanzar en el dominio de las competencias didácticas.

\section{Fundamentación Metodológica}

Los hallazgos encontrados en los proyectos citados, estudios y aportaciones a Congresos y programas de actualización docente, se han llevado a cabo mediante un sistema integrado de métodos, con plena complementariedad entre:

- Didácticos y heurísticos.

- Cuantitativos y cualitativos.

- Experiencias de docentes, expertos y estudiantes.

- Amplitud de ámbitos de investigación en empresas, universidades y escenarios emergentes.

- Aportaciones de diversos países y observatorios en investigación de la docencia.

Todos estos métodos y ámbitos han constituido un diseño ad hoc de instrumentos de recogida de datos. La metodología de encuesta se ha aplicado con el diseño de cuestionarios valiosos, que estiman la génesis, desarrollo y consolidación de las competencias valoradas. La visión cuantitativa se ha ampliado con escalas de observación, documentos ad hoc y análisis de incidentes críticos. La perspectiva cualitativa ha creado nuevos portafolios, cuadernos de campo, observaciones participantes y complementariedad de fuentes de los agentes del proceso de enseñanza-aprendizaje.

De esta manera se ha estimado la pertinencia de las competencias emergidas en el proceso formativo, mediante el uso integrado y el desarrollo de cuestionarios, narrativas, grupos de discusión, diálogos emergentes, fuentes documentadas, vivencias compartidas, etc. A la vez que se ha intensificado los estudios de caso, en la opción de múltiples casos y de problemas compartidos. ${ }^{17}$ Tashakkori y Teddlie, ${ }^{18}$ Trahar.

\section{Análisis del proceso formativo: Complementariedad entre narrativa, grupos de discusión y cuestionarios}

La evaluación de la práctica profesional ha de estudiarse y complementarse con estudio de casos como los realizados y ampliados con

17 TASHAKKORI, Abbas y TEDDLIE, Charles. (2002): "Handbook of mixed methods in social and behavioral research". Londres, Sage publications, p.570.

${ }^{18}$ TRAHAR, Sheila. (ed.) (2009): "Narrative research on learning comparatives and international perspectives". Cambridge, University press, p. 68. 
la auto y coobservación, al menos en parejas, de los procesos formativos realizados. La continuidad de este trabajo con procesos de observación compartida, pondrá de manifiesto si se han alcanzado los objetivos previstos y si el profesorado se implica vitalmente en la construcción del conocimiento profesional, ampliando el análisis de las tareas y la mejora continua de la práctica docente.

La matriz de datos obtenida nos ha propiciado los hallazgos más representativos en las competencias más relevantes para los numerosos docentes consultados, así sintetizamos del estudio en colaboración entre varios países, la selección de un caso, la Universidad a Distancia, y se ha constatado que se aceptan el conjunto de las referidas en el marco introductorio, pero esta universidad subraya algunas competencias nucleares para los docentes de la sede central y la complementariedad, altamente valorada por los docentes tutores de centros asociados en varias competencias docentes, como la competencia tutorial, la tecnológica-diseño de medios y la planificadora que en esta universidad alcanzan valoraciones relevantes.

Los métodos aplicados son coherentes con las principales aportaciones e investigaciones citadas así, ${ }^{19} \mathrm{Kallioinen}$, subraya que para formar a los estudiantes en las competencias genéricas ha de realizarse un destacado estudio de los resultados de los aprendizajes que lleven a su logro y se requiere que el profesorado facilite a los estudiantes el conocimiento claro y el alcance de los objetivos que se pretenden en el EEES.

Entre los términos más empleados en el proceso de la discusión de la formación y desarrollo de las competencias docentes, es este vocablo el de mayor reconocimiento: "las competencias", que ha sido seguido de la comunicativo-lingüística influida por el medio. Esta frecuencia en el conjunto de los siete grupos de discusión representa una clara situación de predominio, frente al resto de los términos o competencias más frecuentes, así en contraposición a la lingüística, ha sido la competencia de diseño de medios, seguida de la evaluadora las menos empleadas y explicitadas en los grupos de discusión (focales).

El diseño del cuestionario se ha llevado a cabo mediante el proceso de validez de contenido, colaborando los expertos (17), en la construcción. La complementariedad con otros cuestionarios y la valoración de su pertinencia al entorno español se ha logrado mediante un meticuloso análisis de las dimensiones de las competencias y de las preguntas constitutivas.

${ }^{19}$ KALLIOINEN, Outi. (2010): "Defining and comparing generic competences in higher education" en: European educational research journal, Vol. 19 No. 1, Oxford. Symposium Journal, pp. 56-68. 
Las competencias se han estimado por los docentes participantes en la reflexión y han sido consideradas en dos modalidades de valoración:

a) Preguntas que han facilitado la ponderación de las más adecuadas y del sentido que cada competencia tiene para los docentes participantes y han sido respondidas en una escala de 1 a 6 .

b) Situar el conjunto de las competencias y ofrecerlas a los implicados para que llevaran a cabo un proceso de "ordenación" de la significatividad y relevancia de cada una para responder a una óptima docencia universitaria.

La fiabilidad o confiabilidad de las dimensiones del cuestionario fue realizada mediante la obtención del alfa de Cronbach, alcanzando en todos los bloques/competencia puntuaciones superiores a 0.90 , lo que implica una significativa fiabilidad.

Los sujetos implicados en la investigación correspondían a las diversas facultades de la universidad, prioritariamente: Educación, Economía, Ingeniería, Derecho, que emitieron un gran número de los 230 cuestionarios, a la vez que los docentes-tutores de los centros asociados: Talavera, Canarias, Segovia, Pontevedra, Tarrasa, etc., complementaron en un conjunto próximo al centenar, los cuestionarios.

Los grupos de discusión, tanto en las facultades de Educación, Economía e Ingeniería, ofrecieron amplias propuestas y singulares aportaciones al reto de la formación de las competencias docentes. Eran heterogéneos en sexo, edad, experiencia y valoración del EEES, y los restantes grupos de discusión se corresponden con los tutores de los citados centros.

Los hallazgos obtenidos en los diferentes instrumentos de recogida de datos se caracterizan por:

- Amplitud y pertinencia de los emergidos en los cuestionarios, diseñados ad hoc y que constituyen una de las aportaciones que el equipo ha propiciado a la comunidad científica.

- Singularidad y relevancia de los hallazgos explicitados en los grupos de discusión. Estos hallazgos implican que los docentes tienen un destacado interés por entender, dominar y compartir modelos de desarrollo profesional, basados en las competencias básicas.

- Perseverancia de datos e interés de los investigadores en el trabajo de campo, que permitió alcanzar nuevas mejoras y proponer a los participantes una línea de estudio, coherente con las más valiosas aportaciones de la investigación en torno a las competencias docentes.

- Novedad, dado que el pensamiento y la práctica docente universitaria está más centrada en el avance y consolidación de los saberes de las disciplinas, que se manifestó estimando las competencias, reconociendo 
el contenido o saber universitario como un componente nuclear de la competencia.

La presentación de los datos y los hallazgos más relevantes del estudio procedemos a realizarlo de forma sintética, a partir de la identificación de cada competencia y de los datos obtenidos en las técnicas de recogida más pertinentes. En coherencia con el diseño de las matrices elegimos las competencias esenciales y procedemos a su análisis atendiendo a los hallazgos de la pluralidad de cuestiones, sintetizando la media y retomando cuando es conveniente, la moda del conjunto de respuestas, tratados los datos con el programa SPSS y siguiendo el proceso más característico, a la vez que retomamos los aspectos cualitativos y complementamos la síntesis de frecuencias en cada competencia, mediante la aplicación del programa Nudis, ajustando y contrastando las frecuencias con un segundo nivel de análisis, con la selección de proposiciones y evidencias de expresiones transcritas y recogidas en los abundantes textos de esta recopilación.

Completamos este análisis integrador de frecuencias y de frases paradigmáticas con un método esencial para descubrir el sentido formativo otorgado a las competencias por los docentes participantes en el estudio y que es extraído de varias fuentes: preguntas abiertas del cuestionario, ordenación y estructuración de las competencias, textos representativos de los grupos de discusión, selección de expresiones prototípicas, sintetizadas en las voces más relevantes de los protagonistas.

\begin{tabular}{|c|c|c|c|}
\hline Competencia & Cuestionario & Grupo de discusión & Narrativa \\
\hline $\begin{array}{l}\text { Investigación e } \\
\text { innovación de la } \\
\text { docencia }\end{array}$ & $\begin{array}{l}\text { Las respuestas a los } \\
\text { cuestionarios sitúan la } \\
\text { estructuración y relevancia } \\
\text { de esta competencia en un } \\
\text { lugar medio-superior }\left(3^{\prime} 83\right) \text {, } \\
\text { destacando la innovación, } \\
\text { que se incrementa en el } \\
\text { conjunto de las preguntas } \\
\text { que la definen y subrayan } \\
\text { la pertinencia de esta } \\
\text { competencia para los nuevos } \\
\text { planes, innovación(4.14). }\end{array}$ & $\begin{array}{l}\text { Los implicados en los } \\
\text { grupos manifiestan su } \\
\text { aceptación: "La docencia } \\
\text { es un mundo en cambio } \\
\text { y ha de orientarse a la } \\
\text { mejora permanente y } \\
\text { conseguir que todos los } \\
\text { implicados la valoren en } \\
\text { alto grado". El análisis } \\
\text { de contenido evidencia } \\
\text { la voz investigación con } \\
\text { una frecuencia (85). }\end{array}$ & $\begin{array}{l}\text { La voz de los } \\
\text { participantes en el } \\
\text { conjunto de frases } \\
\text { y de los textos se } \\
\text { concreta en: } \\
\text { "La investigación de } \\
\text { nuestra docencia ha } \\
\text { de ocupar un lugar } \\
\text { apropiado para } \\
\text { responder a los } \\
\text { retos del EEES". }\end{array}$ \\
\hline $\begin{array}{l}\text { Integración e } \\
\text { implicación en } \\
\text { la sociedad del } \\
\text { conocimiento }\end{array}$ & $\begin{array}{l}\text { La sociedad del } \\
\text { conocimiento es el } \\
\text { verdadero contexto de los } \\
\text { docentes y su estimación es } \\
\text { la media alcanzada: } 4.5 \text { y la } \\
\text { moda (4), que representan } \\
\text { el logro de valores } \\
\text { superiores. }\end{array}$ & $\begin{array}{l}\text { La sociedad del } \\
\text { conocimiento alcanza } \\
\text { una frecuencia de (32), } \\
\text { en el conjunto de las } \\
\text { valoraciones ocupa el } \\
\text { tercer puesto menos } \\
\text { valorado, pero se } \\
\text { obtiene una presencia } \\
\text { suficiente entre los } \\
\text { valores }\end{array}$ & $\begin{array}{l}\text { "El profesorado } \\
\text { se enfrenta a } \\
\text { una sociedad } \\
\text { transformadora, } \\
\text { dinámica y llena } \\
\text { de cambios, } \\
\text { que marcan un } \\
\text { escenario generador } \\
\text { de sentido y de gran } \\
\text { impacto para la vida } \\
\text { universitaria" }\end{array}$ \\
\hline
\end{tabular}


Desarrollo

del sistema

metodológico.

(desarrollo

de estrategias

didácticas)

Valoración de

las dimensiones

europeas (EEES)

Planificación

del proceso de

enseńanza

aprendizaje.
Los valores alcanzados en el cuestionario la sitúan como una de las más destacadas. Media: 4.44 y Moda: 5.0, es considerada muy relevante para la mejora de la docencia y se instala en una situación muy destacada especialmente en los contextos plurilinguísticos.

El sistema metodológico es considerado una competencia nuclear y de gran trascendencia, asíse obtiene una media: $4.8 \mathrm{y}$ una Moda. 5.0. que la sitúan en un punto álgido.

El compromiso de los docentes con el EEES se está incrementando y alcanza el valor de $4.04 \mathrm{y}$ una Moda: 4.0 pero no se encuentra entre las más elevadas.

Esta competencia alcanzó una Media: 3.98 y una Moda. 4.0.

La valoración se encuentra en un lugar medio-alto del conjunto de las estimadas.

Esta competencia es considerada relevante para el profesorado de educación a distancia. La media obtenida: $4.15 \mathrm{y}$

Diseńo de medios la Moda: 4.0 que la sitúa en un nivel cercano a las valoraciones altas.
La frecuencia obtenida en esta competencia es muy elevada (85), que representa una alta estimación para el profesorado y sintetiza el dominio del arte y la oratoria entre docentes y discentes.

La metodología se considera central y logra una frecuencia de (80), que la sitúa entre las más estimadas.

La voz EEES es nueva y representa una presencia de cierto impacto, asíla frecuencia alcanzada es (65) conscientes del reto que representa y del momento de incidencia.

La presencia del término planificación del saber y del conjunto de actuaciones didácticas, presenta una frecuencia de (65) que le sitúa hacia la mitad de las frecuencias obtenidas.

La estimación en el conjunto de las voces expresadas en los grupos la sitúa baja (24) que representa una disonancia con la puntuación alcanzada en el cuestionario. Hemos de diferenciar la voz "medio" de "Diseńo de medios didácticos".
"La tarea docente en sociedades interculturales ha de avanzar en el dominio lingüístico y en la pluralidad de formas de comunicación entre docentes y estudiantes".

"El profesorado marca una diferencia como profesional por el dominio, perseverancia y calidad del sistema metodológico que aplica en el proceso de enseńanzaaprendizaje".

Las voces que se centran en "El EEES es una realidad, que se nos impone y ante la que hemos de reaccionar y actuar con inteligencia y nuevas dosis de creatividad"

"La acción formativa a distancia requiere un proceso de previsión y anticipación rigurosa y de fundamentación de las finalidades medios y tareas más relevantes a llevar a cabo"

El profesorado ha de ser experto en el "diseńo de medios y construir una línea creativa de presentación, uso e innovación continua de los mismos, dado que son necesarios para una educación mediada". 


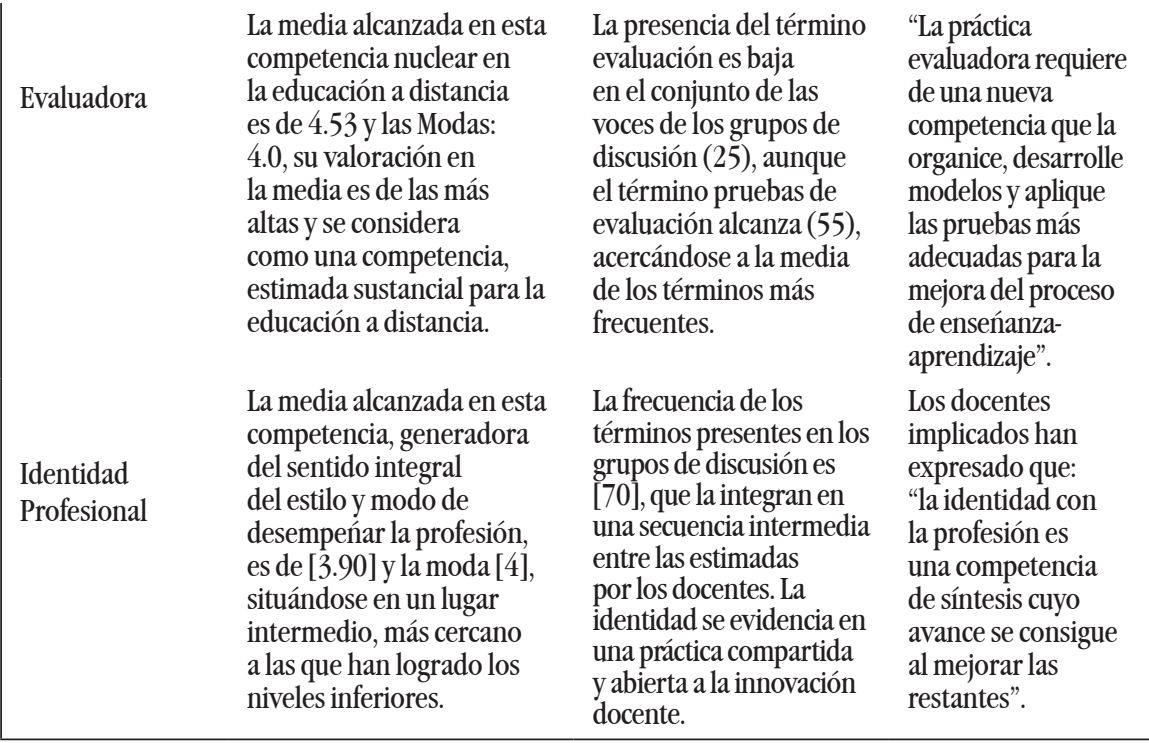

El análisis y contraste de las competencias nos evidencia que la totalidad de ellas, en una escala de 1 a 6, han superado la puntuación de (3.8) y la mayoría supera el (4.2) llegando la mitad a sobrepasar el (4.3) y sólo tres se sitúan debajo del (4.0).

Los análisis de datos nos evidencian un claro avance del profesorado en las competencias docentes citadas, a la vez que en la complementariedad metodológica se observan algunos aspectos que valoramos, singularmente al profundizar en las estimaciones otorgadas a cada una. Los contrastes se dan tanto entre los resultados alcanzados en el cuestionario, como en las frecuencias obtenidas en los grupos de discusión, así constatamos:

La presencia o mayor frecuencia de intensidad en los grupos de discusión, corresponde a las siguientes competencias: Profesionalización o identidad profesional (85); Investigación (85); Institucional o de cultura institucional (65).

En el conjunto de estas competencias encontramos, que las más ligadas a los procesos formativos y al avance en la calidad del acto de enseñanza-aprendizaje alcanzan en los grupos de discusión una menor frecuencia, así la secuencia de menos a más es: medios didácticos (24), evaluación (25), conocimiento práctico (32), innovación (34); sin embargo forman parte del universo de preocupaciones del profesorado y tienen incidencia en la práctica formativa.

Al indagar las estimaciones alcanzadas en cada competencia en el cuestionario, destacamos los siguientes valores de media:

- Integración e implicación en la sociedad del conocimiento (4.54)

- Evaluación del proceso de enseñanza-aprendizaje (4.53) 
- Comunicativa-lingüística (4.44)

- Tutoría (4.43)

Seguidas de:

- Diseño y usos de medios didácticos (4.15)

- Innovaciones didácticas (4.14)

- Valoración de las dimensiones del Espacio europeo de Educación Superior (EEES), (4,04).

Así mismo las menos valoradas han sido:

- Planificación de la docencia y los saberes (3.98)Adquisición de la identidad profesional (3.90)

- $\quad$ Líneas de investigación en docencia (3.83)

Esta secuencia alcanzada en los valores del cuestionario, evidencia algunos contrastes dignos de resaltar, así destacamos:

- La identidad profesional y la investigación alcanzan una elevada frecuencia en los grupos de discusión, especialmente la primera; en consecuencia señalamos que hemos de continuar profundizando en este contraste, considerar estas ordenaciones y en tal ordenación, la construcción de una línea de investigación de la docencia en las universidades, que alcanza la tercera posición y la reflexión acerca de la identidad profesional, la segunda menos valorada.

- Así mismo, la competencia evaluadora y el diseño de medios presentan una divergencia, dado que la evaluadora obtiene la segunda media (4.53) y el diseño de medios (4.41); mientras que en el nivel de frecuencia de los grupos de discusión se señalan como las más bajas (25) y (24) respectivamente.

\section{Análisis y discusión de estos datos}

Las competencias docentes señaladas nos llevan a una nueva reflexión, que nos facilita el contraste de datos, a saber, ¿Qué competencias docentes, son esenciales para responder a la formación de los estudiantes en las genéricas/básicas, específicas y profesionales)?. El conjunto de competencias, emergidas en esta investigación, destaca por su clara aceptación por los docentes de la UNED, especialmente algunas de ellas como la función tutorial, la identidad profesional, y el sistema metodológico y el diseño de medios, para el profesorado-tutor, colaboradores imprescindibles del modelo presentado, pero que en la opción del enfoque del "Blended-learning", son necesarias para todo docente que desarrolle su práctica profesional en cualquier institución.

La formación de los estudiantes desde el enfoque de las competencias ha significado para el profesorado universitario, la aplicación de la pregunta crucial; ¿Hemos dominado las más adecuadas para el profesorado y estamos capacitados 
para formar a los estudiantes en este enfoque? ¿Conocemos el alcance y retos, que representa esta visión formadora para nuestro desempeño profesional? ¿Forma parte de nuestra línea de investigación e innovación el conocimiento de tales competencias? ¿Estamos convencidos de que es previo el dominio de estas competencias docentes para avanzar cada curso en nuestra identidad y desarrollo profesional? El modo de preguntarnos y de abordar este enfoque marca la principal diferencia, entre la visión académica centrada en el dominio necesario de los saberes, métodos y visiones disciplinares y la tendencia más creativa y enriquecedora que implica capacitarnos para saber, actuar, valorar y sentir el gran reto de la formación en competencias docentes y discentes.

Los datos obtenidos y la profunda implicación de una amplia muestra próxima a 250 colegas, así como la pluralidad y complementariedad metodológica, avalan los hallazgos y ratifican la validez y confiabilidad de las dimensiones o competencias seleccionadas, de las preguntas que han identificado e implicado a cada docente en la estimación de las competencias y la gran riqueza del proceso central: Ordenación de las competencias, emergidas y consolidadas previamente, mediante el método de Delphi, apoyado en la relevancia de los investigadores expertos y profesionales, que "nominaron, formularon y dieron un nuevo enfoque a las competencias docentes" más relevantes en la sociedad del conocimiento, en el nuevo EEES y en el desafío de la globalización universitaria.

Estas competencias han de orientarse a formar a los estudiantes universitarios en aquellas competencias que mejor identifiquen su ser y hacer, como tales estudiantes de educación superior. El dominio de las competencias por los estudiantes, demanda las correspondientes del profesorado, por su cercanía, profundizando en las pertinentes competencias docentes, pero nos hemos de cuestionar, si es factible que el profesorado que no haya tomado conciencia del proceso a seguir para formar en el saber y hacer de las propias competencias. ¿Se encuentra capacitado para a su vez, preparar a los estudiantes en el dominio de tales competencias, como futuro profesional y en las tareas socio-laborales específicas?

El profesorado ha de tomar conciencia del conjunto de competencias que le caracterizan y que la sociedad del conocimiento, las nuevas profesiones y la pluralidad social demanda, conscientes del papel cambiante y de las grandes demandas de los estudiantes, las organizaciones productivas y un mundo en transformación permanente. Así los modelos formativos serán más directos y han de plantear elementos acordes con el tipo de preparación que los estudiantes y la sociedad necesitan en este siglo; de acuerdo con el ritmo de cambio y de las respuestas que hemos de ofrecer, no sólo con legitimidad y gran fundamentación epistemológica, sino con nuevas evidencias de prácticas fecundas, y orientadas a la capacitación de los estudiantes y al desarrollo de las organizaciones. 
Los datos de la investigación propician un camino, que cada docente y equipo han de madurar, readaptar y tomar conciencia del plan de acción que de modo individual y autónomo, además de colaborativo e institucional hemos de aportar. Estos hallazgos subrayan que se ha de asumir y reconocer que este enfoque de desarrollo profesional y de dominio, profundización y mejora de las competencias acordadas es pertinente, en consecuencia ha de actuar para profundizar en las mismas y compartir con los colegas el itinerario de formación y avance profesional que asuma.

\section{CONCLUSIÓN}

Los objetivos pretendidos son estimados en su proceso y hemos de consolidar tanto el mapa de las competencias más relevantes para una mejora de la docencia, cuando un proceso de desarrollo profesional que comprometa al profesorado en la consolidación y transformación de lo más pertinente para alcanzar una cultura de indagación y de transformación de la práctica profesional.

La conclusión más relevante es que se han identificado un conjunto de competencias de gran impacto en la mejora de la docencia universitaria, aglutinando dos grandes bloques, las que atañen a los retos de la sociedad del conocimiento, el nuevo escenario europeo (EEES), la comunicación abierta e intercultural, los procesos de investigación e innovación de la docencia universitaria; que en su conjunto representan los grandes cambios a los que los docentes universitarios han de atender.

Un segundo bloque, que se centra en los aspectos característicos de la mejora de la práctica docente y que tiene un acentuado perfil didáctico como: planificación del proceso de enseñanza-aprendizaje, tutoría, sistema metodológico y estrategias didácticas, diseño y uso de medios didácticos y evaluación del proceso de enseñanza-aprendizaje, que contribuyen a configurar una mejor imagen de la docencia y propician la satisfacción profesional, sintetizada en la metacompetencia de identidad profesional.

La complementariedad de métodos y acciones desarrolladas avalan la pertinencia de estas competencias, dado que la menos valorada en el cuestionario alcanzó una media de (3.83), que supera el valor medio determinado, a la vez, que la moda en más de la mitad de las competencias obtiene el valor (5.0), si a ello añadimos las voces explicitadas en las narrativas, grupos de discusión e intercambio entre expertos, se obtiene una adecuada estimación. Otras investigaciones y comunidades didácticas, evidencian que el Mapa de Competencias diseñado es de gran valor y se asienta en la discusión y concordancia con otros trabajos de diversos investigadores ${ }^{20}$ (Zabalza, Villar y Alegre, De la Hoz, Medina y cols.).

${ }^{20}$ ZABALZA, Miguel Ángel. (2006): Óp., cit, p. 39. DE LA HOZ, Gustavo. (2009): Óp., cit, p. 58. VILLAR, Luis y ALEGRE, Olga. (2009): Óp., cit, p. 40.

MEDINA, Antonio y cols. (2006): Óp., cit, p. 76. 
Los objetivos expresados al inicio de este artículo han sido alcanzados en su globalidad y se ha constatado su logro mediante los datos y bases documentales trabajadas.

\section{FUENTES}

Congreso Internacional sobre las nuevas bases para el Diseño de los Planes de Estudio en el EEES y su incidencia en el diseño educativo. 2009. Madrid, Ed. Universitas.

Congreso Internacional sobre Investigación e Innovación de la Docencia universitaria en el EEES. (2010). Madrid, Ramón Areces.

Red Iberoamericana de Investigación de la Calidad de la Educación Superior. (2010). RIAICES, (actualmente, nueva URL 2012).

Proyecto de desarrollo futuro en el Espacio Europeo de Educación Superior. (2007). Madrid, Ed. Ministerio de Universidades e Innovación (edición on line).

\section{REFERENCIAS}

DE LA HOZ, Gustavo. (2009): Los médicos y la Educación Médica: Una mirada desde la teoría sobre la formación de competencias socio - profesionales en el profesorado universitario. Barranquilla, Universidad Libre.

DOMÍNGUEZ, María Concepción; MEDINA, Antonio; CACHEIRO, María Luz. (2010): Investigación e innovación de la docencia universitaria en el EEES. Madrid, Editorial Universitaria Ramón Areces.

KALLIOINEN, Outi. (2010): "Defining and comparing Generic Competences in Higher Education", en: European Educational Research Journal. Vol 19, No 1. Symposium Journal.

LE BOTERF, Guy. (2010) : Construire les compétences individuelles et collectives: Agir et réussir avec competence. París, Eyrolles.

MEDINA, Antonio y DOMÍNGUEZ, María Concepción. (2008): "La formación integral, base del desarrollo de las comarcas". Madrid, Universitas.

MEDINA, Antonio (ed.). (2009): "Formación y desarrollo de las competencias básicas”. Madrid, Universitas.

MEDINA, Antonio. (2010): Formación y desarrollo de competencias básicas y profesionales, en: Investigación e innovación de la docencia universitaria en el EEES. DOMÍNGUEZ, María Concepción; MEDINA, Antonio, y CACHEIRO, María Luz. (eds.). Madrid, Editorial Universitaria Ramón Areces.

MEDINA, Antonio. (2003): "Modelos de evaluación de la calidad de las instituciones universitarias". Madrid, Universitas.

MEDINA, Antonio y cols. (2006): "Formación y evaluación de las competencias del profesorado”. Madrid, Ministerio de Universidades e Investigación (Proyecto MOEES).

MEDINA, Antonio, SEVILLANO, María Luisa y DE LA TORRE, Saturnino. (Coords.) (2009): "La Universidad del S. XXI”. Madrid, Universitas.

MEDINA, Antonio. (2008): "La convivencia en las escuelas interculturales: el clima social colaborativo y su incidencia en la mejora del acoso escolar", en: "Itinerarios metodológicos en la investigación social. GUILLÉN, Manuela y VALENZUELA, Blanca A. Sonora, Hermosillo, Universidad de Sonora. pp. 179-204. 
MEDINA, Antonio y DOMÍNGUEZ, María Concepción. (1990): "Formación del profesorado en una sociedad tecnológica”. Madrid, Cincel-kaplusz.

MEDINA, Antonio y DOMÍNGUEZ, María Concepción. (2006): “Los procesos de observación del prácticum: Análisis de las competencias", en: Revista Española de Pedagogía, LXIV. No 233. Madrid, España.

MEDINA, Antonio y DOMÍNGUEZ, María Concepción. (2007): "La formación de las competencias profesionales mediante el empleo de la plataforma de investigacion en docencia universitaria", IV jornadas ICE, Alicante 4-5 julio.

MEDINA, Antonio y DOMÍNGUEZ, María Concepción. (2008): Los procesos reflexivos como base de formación del profesorado, en: CARDONA, José. Cuestiones en torno a la formación y desarrollo profesional de los profesores, Madrid, Sanz y Torres.

MEDINA, Antonio y RODRÍGUEZ, Ana. (1997): "Programas de formación inicial del profesorado en la universidad", en: Actas V Congreso Interuniversitario de Organización de Instituciones Educativas. Madrid, 10-13 de noviembre.

MEDINA, Antonio; DOMÍNGUEZ, María Concepción; SÁNCHEZ, Cristina (2010): "Formación de las competencias socio-profesionales desde la función tutorial", en: III Jornadas de Redes. Madrid, UNED.

MEDINA, Antonio; DOMÍNGUEZ, María Concepción; SÁNCHEZ, Cristina. (2009): "Formación de las competencias de los discentes, mediante un diseño integrado de medios", en: Revista ECCOS, V. 10. N 2, Sao Paulo, Ed. UNIOVI.

MEDINA, Antonio. (ed.) (2003): “Autoevaluación de la docencia universitaria", en: Modelos de evaluación de la calidad en instituciones universitarias. Madrid, Universitas.

TASHAKKORI, Abbas y TEDDLIE, Charles. (2002): "Handbook of mixed methods in social and behavioural research". London, SAGE Publications.

TRAHAR, Sheila (ed.). (2009): "Narrative research on learning comparatives and international perspectives". Cambridge, University Press.

VILLAR, Luis Miguel. (2004): "Programa para la mejora de la docencia universitaria". Madrid, Pearson Educación.

VILLAR, Luis Miguel y ALEGRE Olga. (2009): "Investigación evaluativa de un título propio: Mater EDECAR en la diversidad", en: Revista Enseñanza. Ed. Universidad de Salamanca.

VILLAR, Luis y ALEGRE, Olga. (2010): "El plan de desarrollo profesional docente universitario (D.P.D.U.) en las islas canarias mediante plataforma en línea". Las Palmas de Gran Canaria. URL: http://www.colombiaaprende.edu.co/html/ mediateca/1607/articles-108365_archivo.pdf (última consulta: 29/3/2012).

ZABALZA, Miguel Ángel. (2006): "Competencias docentes del profesorado universitario. Calidad y desarrollo profesional”. Madrid, Narcea.

ZABALZA, Miguel Ángel. (2006): "La Universidad y la docencia en el mundo de boy”. Bogotá, Multimedias.

MEDINA RIVILLA, Antonio; DOMÍNGUEZ GARRIDO, $\mathrm{M}^{\mathrm{a}}$ Concepción; RIBEIRO GONÇALVES, Fernando. (2011):

"Formación del profesorado universitario en las competencias docentes" en: Revista Historia de la Educación Latinoamericana Vol. 13 No. 17, Tunja, Universidad Pedagógica y Tecnológica de Colombia, RUDECOLOMBIA, SHELA- HISULA. pp. 119-138 